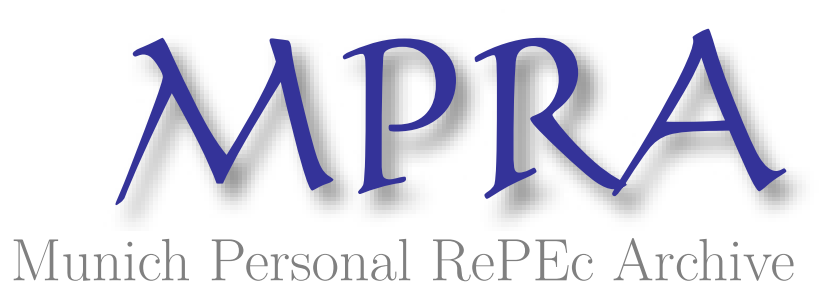

\title{
Physical Modeling of economic systems: Classical and Quantum Economies
}

Kondratenko, Anatoly

2005

Online at https://mpra.ub.uni-muenchen.de/10452/

MPRA Paper No. 10452, posted 30 Oct 2008 02:06 UTC 


\section{EXCELL GROUP}

INNOVATIVE CONSULTING COMPANY

ANATOLY V. KONDRATENKO

\section{PHYSICAL \\ MODELING OF ECONOMIC SYSTEMS}

CLASSICAL AND QUANTUM ECONOMIES 


\section{Introduction}

In the market economy major active players (or agents, or subjects), i.e. buyers and sellers of goods and commodities, behave to a certain extent in a deterministic way thus subordinating their behavior in the market to some strict economic laws. The fact that these laws are of a descriptive nature, and they have not yet been expressed in a precise mathematic language, is not of key importance in this case.

Every rational player or market subject acts in the market in accordance with a strict rule of obtaining maximum profit or benefit or some other criterion of optimality for him or her and in this respect market economic systems resemble physical systems where all players, members, and elements of the system act also in accordance with some principles of maximization.

The main drive of our research is that we assume general possibility to develop dynamic models for relatively simple market economic systems consisting of an economic subsystem or simply an economy with a certain number of buyers and sellers and its external environment with certain interactions between economy agents, and between the economy and environment. In this case, it is supposed that it can be performed by the analogy with the method of developing theoretical models of physical systems consisting of a system of interacting material particles in the external fields or external environment [1, 2]. Moreover, it is assumed that equations of motion derived for physical systems in the physical space are quite a good initial approximation for equations of motion of modeled economic systems in some price space.

Let's give the following reasons to substantiate such an approach. Let $p(t)$ be a trajectory of movement of the market subject in a price space, in other words, it is price $p$ of a commodity set for by the subject at a moment $t$. By setting prices this way, buyers and sellers who act as homo negotians (a negotiating man) in a physical modeling aim at maximum satisfaction of their strive for profit, i.e. for such price at which interests of both buyers and sellers and, considering external environment and the whole system in general, are satisfied in an optimal way. It is here that one can see similarity of the movement of economy (described by 
trajectories $p(t))$ to the moving of material particles of the physical system (described by trajectories $x(t)$ ), which is also subject to a certain principle of maximization. This is what our confidence in conceptual possibility of developing a dynamic theory of economic systems in a price space by the analogy with a dynamic theory of physical systems in physical space is based on. We also admit that in spite of the difference between the qualities of the physical and price spaces along with the qualities of economic and physical systems, the reasonable starting point in the study of economic systems dynamics are equations of motion for a physical prototype, i.e. the type of equations of both systems in their spaces will be the same, though the essence of parameters and potentials in them will be completely different. It is a usual case in physics when one and the same equation describes different systems. For example, the equation of harmonic oscillator describes motion of both simple pendulum and electromagnetic wave. In other words, formal similarity of the equations does not mean equality of the systems which they describe.

In physics a wide experience in calculating physical systems of different degree of complexity with different interaction between members of the system and the system with external environment has been accumulated. It makes sense trying to find a way to use these achievements in finding solutions of economic problems. In case such an activity were successful, there would appear an opportunity of numerical research of the influence of both different internal and external factors on the behavior of each economic agent and on the results of activity of the whole economic system with the help of computer calculations of physical models. Economics (more exactly - theoretical economics) would obtain the most powerful research device the opportunities of which could be compared with the result of the discovery of equations of motion of the physical systems.

The logic of present research is the following. At the first stage of designing a physical model and deriving equations of motion of economic systems from the first principles, or ab initio, we first make all approximations and assumptions required for deriving equations of motion of physical systems in the course of theoretical physics by Landau and Lifshitz [1, 2], then we obtain economic systems equations which are similar to physical systems equations in form and are considered by us as starting or initial approximation for a physical model of the economic system. At the second stage massive model calculations are carried out, their results are compared with experimental or test results, in other words, with the data of empirical economics. At the same time, some optimum values of model parameters and potentials are obtained and 
the applicability limits of the given initial approximation are estimated. At the third stage suppositions are estimated comparing calculation results and experimental data, and more precise and appropriate optimization principles and corresponding equations of motion are found.

The present book concerns only results of the completed first stage of research at which the main principles of proposed approach to developing physical models of simple market economic systems including economy consisting of several buyers and sellers of certain commodities, and external environment, were developed. 


\section{Main elements of the physical models}

Main elements of our physical models of the economic systems are shown schematically in Fig. 2.1. A large sphere covers a market subsystem or simply economy consisting of active market subjects: buyers who have financial resources and a desire to buy goods or commodities, and sellers who have goods or commodities and a desire to sell them. They are the buyers and sellers who form demand and supply in the market. Small dots inside the sphere denote buyers, big ones denote sellers. Cross-hatched area outside the sphere represents external environment including the state, society and other institutions. The whole economic system is located in some commodity-and-price space which will be discussed below. It is evident that all elements of the system influence each other: buyers compete with each other in the market for commodities; sellers compete with each other for buyers.

The same way buyers and sellers interact with each other thus influencing each other's behavior. External environment influences all economic agents. In other words, all economic agents are influenced by external environment and interact with each other.

In order to develop a physical model of the economic system, it is necessary to learn to describe exactly in a mathematical way both movement (behavior) of each economic agent, i.e. buyers and sellers, and interaction with each other, and in the end to derive equations of motion

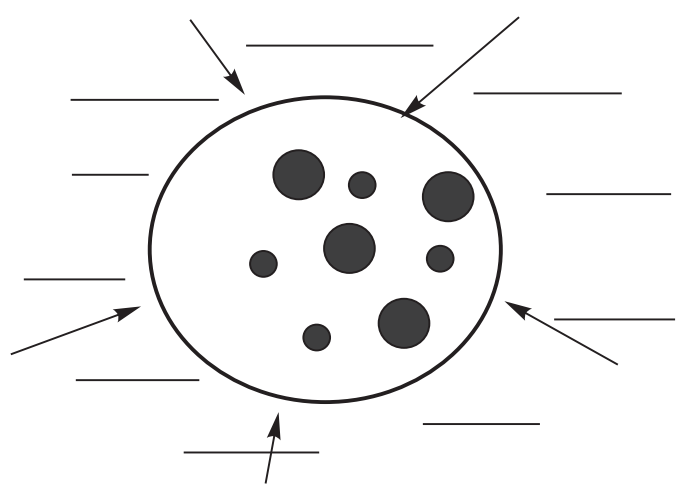
which show dynamics or movement, or evolution of the system in time.

Fig. 2.1. Physical model scheme of an economic system: economy consisting of buyers (small dots) and sellers (big dots) which is under the influence of external environment beyond the economy (covered by the sphere). 


\section{Price space}

To show the movement or dynamics of economy it is necessary to introduce a space in which this movement takes place. As an example of such space we choose a commodity-and-price space, or to be more exact simply a price space created by the analogy with a common physical space, where, though, we choose prices $p_{i}$ of the $i$-th item of commodities as coordinate axes: $i=1,2, \ldots, T$, where $T$ is the amount of items of commodities. In case there is one commodity, the space is one-dimensional, i.e. it is represented by one line; the coordinate system for one-dimensional space is shown in Fig. 3.1. The distance between two points in one-dimensional space $p^{\prime}$ and $p^{\prime \prime}$ is determined as

$$
\left|p^{\prime}-p^{\prime \prime}\right|=\sqrt{\left(p^{\prime \prime}-p^{\prime}\right)^{2}} \text {. }
$$

In case there are two commodities, the space is a plane; the coordinate system is represented by two mutually perpendicular lines (see Fig. 3.2), and the distance between two points $p^{\prime}$ and $p^{\prime \prime}$ is determined as

$$
\left|p^{\prime \prime}-p^{\prime}\right|=\sqrt{\left(p_{1}^{\prime \prime}-p_{1}^{\prime}\right)^{2}+\left(p_{2}^{\prime \prime}-p_{2}^{\prime}\right)^{2}} \text {. }
$$

After the analogy of it we can build a space of any dimension $T$.

In spite of apparent simplicity, introduction of a price space is of conceptual importance as it allows to describe behavior of market agents in the mathematic language in general. This possibility really exists as setting their own price for commodities any moment of time $t$ is the main function or activity of market agents, and it is, in fact, the main feature or trajectory of agent's behavior in the market.

It is our main goal to learn to describe these trajectories or connected with them distributions of price probability. It is impossible to do this in a physical space, for example: we can thoroughly describe movement or trajectory of a seller with commodities in physical space, espe-

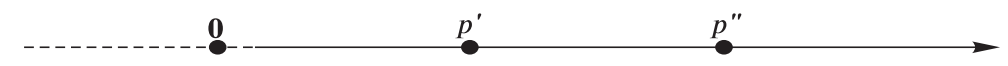

Fig. 3.1. Coordinate system of the one-dimensional price space. 


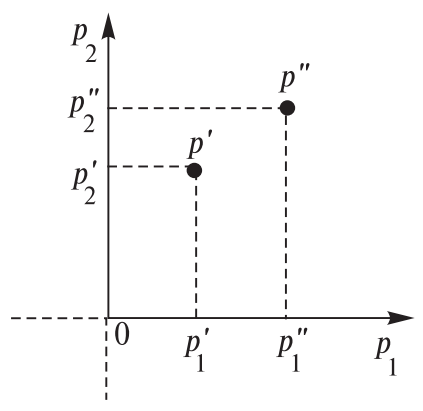

Fig. 3.2. Coordinate system of the two-dimensional price space.

cially if he is in a car or in a spaceship, but it will not have any connection with his attitude to the given commodities and his behavior in this respect (showing his attitude to the commodities by means of his monetary estimation) as an economic agent. Within the problem of describing agents in the market, the role of price as an independent variable, or a coordinate $p$ is considered here as a unique one for market economic systems.

The next step in developing a physical model after selecting a space is selection of a function with the help of which we will try to describe the dynamics of an economy, i.e. movement of buyers and sellers in the price space. Trajectories in coordinate physical space $x(t)$ (classical mechanics), wave functions or distributions of probabilities ||$^{2}$ (quantum mechanics), Green's functions $G$ and $S$-matrices (in quantum physics), etc. are used as such functions in physics. We start with an attempt to develop the model using trajectories in the price space $p(t)$ by analogy with the use of trajectories $x(t)$ of pointlike bodies used in classical mechanics. Such model will in short be called below as a classical model or simply classical economy. 


\section{Classical economy}

According to the above-stated plan of actions in this chapter we could confine ourselves to just writing equations of motion analogous to those obtained in classical mechanics. However, we consider it useful to derive a full row of equations and to make additional comments on our actions. First of all, as we have indicated before, we suppose that according to our approach to classical modeling of economic systems every economic agent, homo negotians, acts in the market not only rationally in his own interests, that is, to gain maximum profit (by negotiating to reach a minimum price for the buyer and a maximum price for the seller) but also reasonably, by leaving his counteragent a chance to get profit from transaction; otherwise, transactions will take place only once while all agents are interested in continuation and stability of their business.

Besides, we suppose that external forces influence market operations positively, establishing common rules of play that favor gaining maximum profit or utility for the whole economic system. Based on these principles we have a firm belief that there is a certain principle of optimization, and its effect results in certain rules of market behavior and certain equations of motion that are followed by all rational or reasonable players spontaneously or voluntarily. In our opinion, it is they who have the leading role in the market.

Let us proceed to deriving equations of motion for classical economy. We follow the same procedure as in classical mechanics [1]. To make calculations easier, we will consider in this paper a single-commodity economy, that is, only movement in one-dimensional price space with variable coordinate $p$. Transition to a multi-dimensional case does not cause principal complications. We will consider that by the analogy with classical mechanics [1], a state of economy comprising $N$ buyers and $M$ sellers and being under the influence of the environment is fully described by establishing all prices $p_{i}$ and their first time $t$ derivatives (price changing rate or velocity of movement) $\dot{p}_{i}=\frac{d p_{i}}{d t}$, where $i=1$, $2, \ldots, N+M$. Let $p$ without subscripts denote set of all prices $p_{i}$ for short, similarly to first and second time derivatives, i.e. for velocities $\dot{p}_{i}$ and ac- 
celerations $\ddot{p}_{i}$. Due to their sense or by definition, equations of motion connect prices, velocities and accelerations. In classical mechanics they are second-order differential equations of time, their solution under assigned conditions at the moment $t_{0}, x\left(t_{0}\right)$ and $\dot{x}\left(t_{0}\right)$, represents the required mechanical trajectories, $x(t)$. We are going to derive similar equations with the same view for our economic system in the price space.

By analogy with classical mechanics we assume that these equations result from a certain principle of maximization (the Hamilton principle in mechanics). Namely, integral $S$ must have the least possible value:

$$
\begin{gathered}
S=\int_{t_{1}}^{t_{2}} L(p, \dot{p}, t) d t, \\
S=0 .
\end{gathered}
$$

The obtained (4.1) and (4.2) lead to equations of motion or Lagrange equations [1]:

$$
\frac{d}{d t} \frac{\partial L}{\partial \dot{p}_{i}}-\frac{\partial L}{\partial p_{i}}=0 \quad(i=1,2, \ldots, N+M) .
$$

Equations of motion represent a system of second-order $N+M$ differetial equations of time $t$ for $N+M$ unknown required trajectories $p_{i}(t)$.

These equations employ as yet unknown Lagrange function or lagrangian $L(p, \dot{p}, t)$ which is to be found on the basis of research or experimental data. We will note that Lagrange functions were used in literature to solve a number of optimization problems of management science [3]. Let us emphasize that determination of Lagrange function is the key problem that can only be solved in practice by making the data of theoretical calculation fit experiment. It can not be done using theoretical methods only. But what we can do quickly is to make the first evident trial step, namely, to assume that to a certain degree of approximation Lagrange function looks like (only looks like) Lagrange function of its physical prototype, a system of $N+M$ material particles with certain potentials. All assumptions made here can be thoroughly analyzed later at the second stage of investigation and left unchanged or made more accurate after comparison with the experimental data. Accomplishment of this stage will naturally require great effort and expenses. As for now, we accept these assumptions and consider that Lagrange functions have the same form as that of its physical prototype but all parameters and potentials of the economic system will be chosen on the basis of economic ex- 
perience, not taken from the physical prototype. We consider that by adjusting parameters and potentials to the experiment we can smooth out negative influence of made assumptions for solutions of equations of motion obtained in such a way.

So, according to our approach, equations of motion in classical economy are nominally identical to those in corresponding mechanical system. However, their constants and potentials will have other essence, other dimensions and other values. A great advantage of classical economies consists in the fact that mathematical solutions of these equations, analytical or numerical, have been found for a great number of Lagrange functions with different potentials, that is why it is of great help to apply them. Let us turn to relatively simple classical economies.

Let us consider a case of economy with a single good, a single buyer, and a single seller, where environmental influence and interaction between a buyer and a seller can be described with the help of potentials. Lagrangian of such an economy has the following form:

$$
L=\frac{m_{1}}{2} \dot{p}_{1}^{2}+\frac{m_{2}}{2} \dot{p}_{2}^{2}-V_{12}\left(p_{1}, p_{2}\right)-U_{1}\left(p_{1}, t\right)-U_{2}\left(p_{2}, t\right) .
$$

In (4.4) $m_{1}$ and $m_{2}$ are certain unknown constant values or parameters of economic agents who are the buyer and the seller respectively. The first two members of equation (4.4) in classical mechanics correspond to kinetic energy, and the rest three ones - to potential energy. Understanding conventional character of these names, we will use them for economy as well. Potential $V_{12}\left(p_{1}, p_{2}\right)$ describes interaction between the buyer and the seller (it is unknown a priori), and potentials $U_{1}\left(p_{1}, t\right)$ and $U_{2}\left(p_{2}, t\right)$ are designed to describe environmental influence on economy. They are to be chosen with respect to experimental data according to dynamics of the modeled economy. Lagrange equations have the following form for such a lagrangian:

$$
\left\{\begin{array}{l}
m_{1} \ddot{p}_{1}=-\frac{\partial V_{12}\left(p_{1}, p_{2}\right)}{\partial p_{1}}-\frac{\partial U_{1}\left(p_{1}, t\right)}{\partial p_{1}}, \\
m_{2} \ddot{p}_{2}=-\frac{\partial V_{12}\left(p_{1}, p_{2}\right)}{\partial p_{1}}-\frac{\partial U_{2}\left(p_{2}, t\right)}{\partial p_{2}} .
\end{array}\right.
$$

This system of two differential second-order equations of time $t$ represents equations of motion for a selected classical economy. According to their form they are identical to the equations of motion of the physical prototype in physical space. In the latter case system (4.5) designates the second Newton's law of classical mechanics: 'product of mass by acceleration equals force'. And quite another matter is that po- 
tentials can be significantly different from the corresponding potentials in physical system. We should mention once again that these potentials are to be discovered for different economies by detailed comparison of results of computation of equations of motion of economies with experimental or research data, in other words, with data of empirical economics. At the initial stage it is natural to try to use known forms of potentials from physical theories, and we are going to do that in future. Let us note that the purchase-sale deal or transaction in the market between the buyer and the seller will take place at the time $t_{c}$ when their trajectories $p_{1}(t)$ and $p_{2}(t)$ intersect: $p_{1}\left(t_{c}\right)=p_{2}\left(t_{c}\right)$. The value $p_{1}\left(t_{c}\right)$ (or $p_{2}\left(t_{c}\right)$ ) is indeed then the price of the good or commodity in the market, i.e. the market price of the good at the time $t_{c}$.

It is interesting that a number of some common features of classical economy with equations of motion (4.5) are common for almost all constants $m_{i}$ and potentials $V_{12}$ and $U_{i}$. Let us consider a case when external potentials $U_{i}\left(p_{i}\right)$ do not depend on time and represent potentials of attraction with high potential walls at the origin of coordinates that prevent economy from moving towards the negative price region, and potential $V_{12}$ depends only on the module of difference of prices of the buyer and the seller $p_{12}=\left|p_{2}-p_{1}\right|$, namely,

$$
V_{12}=V_{12}\left(\left|p_{2}-p_{1}\right|\right) \text {. }
$$

We assume that potential $V_{12}$ describes attraction between the buyer and the seller and has its minimum at the point $p_{12}^{0}$. Then the solution of equations of motion describes movement or evolution of the entire economy as follows: the centre of inertia of the whole system (it is introduced to theory by analogy with the centre of inertia of the physical prototype) moves at a constant rate $\dot{P}$, and the internal movement, i.e. of buyers and sellers relative to each other, represents an oscillation, usually anharmonic, around the point of equilibrium $p_{12}^{0}$. This conclusion is trivially generalized for the case of an arbitrary number of buyers and sellers.

So we get classical economy with the following features:

1. Movement of the centre of inertia at a constant rate signifies that if at some point of time a general price growth rate were $\dot{P}$, then this growth will continue at the same rate. In other words, such type of economy implies that prices increase at a constant rate of inflation (or rate of inflation is constant).

2. Internal dynamics of economy means that economy is oscillating near the point of equilibrium; in this case, economy is found in the equilibrium state only within an insignificant period of time (just as a me- 
chanical pendulum is found in the equilibrium state, that is, at the lowest point, for a short period of time); moreover, rates of changes in relative prices of sellers and buyers are maximal at the point of equilibrium (for the pendulum the rate of movement at the point of equilibrium is also maximal). According to our view, oscillations of economy relative to point of equilibrium $p_{12}^{0}$ represent nothing but business cycles with a certain period of oscillation that is determined by solving equations of motion with specified mass $m_{i}$ and potential $V_{12}$. These results correlate to the Walrasian cobweb model which is well known in neoclassical economic theory [4].

As is seen from the above shown example, physical classical models or simply classical economies deserve thorough investigation, as they happen to become an efficient tool of theoretical economics. However, there are reasons to believe that quantum models where probability conception is used for description of companies' and people's behavior in the market are more adequate physical models of real economic systems. Recall that probability conception was first introduced into economic theory by one of the founders of quantum mechanics, Neumann, in the 20s of the last century [5]. 


\section{Quantum economy}

We know that the principal point in developing a physical model is a selection of function which will help to describe economy dynamics, in other words, movement of buyers and sellers in price space. As such a function in classical economy we have chosen the agent's trajectory in price space $p(t)$, supposing implicitly that behavior or movement of the market agent comes to establishing a price for goods and commodities at every point of time by negotiations or information exchange both between economic agents and agents with external environment. It looks like economic agents adjust their trajectories to each other based on their principal concerns for their own and common profit that leads to some determination in their behavior or movement in price space. In the previous chapter we have received at least non-contradictory classical economies with Lagrange equations as equations of motion.

In the present chapter we will speak of another quantum method of description of movement or behavior of market agents, sellers and buyers, based on the use of probabilistic approach to describe movements of market agents by means of wave functions and related to them distributions of probabilities of sale transactions. Agents with such behavior will be called below as homo oscillans (an oscillating man). The quantum method of description of market agents' movement ( or simply quantum economics) leads to probabilistic mechanism of forming of demand and supply functions and market price at which sale transactions are carried out in the market. Quantum equations of motion and interpretation of their solutions will, of course, be different from those in classical economies. It should be marked that there is an analogous situation in physics.

\subsection{Time-dependent equations of motion of quantum economy}

When deriving equations of motion of quantum economy we will use the same scenario as when we derived equations of motion for classical economy: we make the same assumptions like the ones for equations of motion for physical systems - prototypes in quantum mechanics in 
physical space and so, we get the same equations of motion for economy in price space. Analysis of made assumptions and gradual refinement of model by detailed calculation of equations solutions with different parameters and interaction potentials and by detailed comparison with experimental data or those observed in practice is a subject of our future investigations.

When deriving equations of motion we follow the procedure described in the book by Landau and Lifshitz [2]. First of all, let us assume that economy can be described to a desired degree of accuracy by a certain function in price space $(p)$, where $p$ as before designates a set of all price coordinates of all economic agents with a squared module of this function determining distribution of price value probabilities: ||$^{2} d p$ stands for a probability that economic agents will have prices in element $d p$ of price space. Functions in quantum mechanics are called wave functions. So it is natural to introduce a normalization condition for finite quantities in the whole space:

$$
\int||^{2} d p=1 .
$$

It is also natural to determine that for the economy consisting of two independent economies or subeconomies 1 and 2, total wave function of economy is a product of subeconomy wave functions:

$$
{ }_{21}\left(p_{1}, p_{2}\right)={ }_{1}\left(p_{1}\right){ }_{2}\left(p_{2}\right) .
$$

By analogy with quantum mechanics of physical systems we consider that wave function of economy does not only describe economy behavior at a given point of time $t_{0}$ but also determines its behavior or evolution in all future moments of time. It means that along with some other assumptions that are not considered in this paper, equations of motion for economy can be written in the form of so called wave equation:

$$
i \frac{\partial}{\partial t}=\hat{H} \text {. }
$$

In (5.3) $\hat{H}$ is a certain linear hermitian operator that is called Hamilton operator or a hamiltonian in quantum mechanics. If this hamiltonian is known, then wave equation (5.3) determines wave functions of economy at any point of time. Generally speaking, Hamilton operator is unknown for economic systems, this problem is for future consideration. In (5.3) $i$ is a usual complex variable, and is a certain constant which is also unknown. If in quantum mechanics this is a universal or Plank constant $\hbar$, in quantum economics this is just a parameter which may be different for various economies, and its economic sense will be determined some day. 
Hence, we have obtained the required equations of motion of economy in the wave equation form with unknown constants and Hamilton operator $\hat{H}$. This equation describes economy dynamics in time so let us call it a time-dependent equation of motion, also well-known in physics as time-dependent Schrödinger equation.

\subsection{Stationary equations of motion}

Let us introduce a notion of stationary states for particular case of economies under the influence of constant external fields, i.e. that do not depend on time explicitly. According to definition, stationary states of economy are so called eigenfunctions of Hamilton operator which in this case does not depend on time either. In other words, stationary states are the solution of so called stationary equations of motion (stationary Schrödinger equation in quantum mechanics):

$$
\hat{H}_{n}=E_{n} \quad n .
$$

In equation (5.4) $E_{n}$ denotes eigenvalues of Hamilton operator or energy eigenvalues as they are called in quantum mechanics, or just energy of states $n$. Time-dependent equations of motion are easily integrated for stationary states (or eigenstates):

$$
\begin{aligned}
& i \frac{\partial n}{\partial t}=\hat{H} \quad{ }_{n}=E_{n} \quad n, \\
& { }_{n}=\exp \left(-\frac{i}{-} E_{n} t\right) \quad{ }_{n}(p),
\end{aligned}
$$

where ${ }_{n}(p)$ designates functions of prices only, i.e. they do not depend on time. ${ }_{n}(p)$ are also eigenfunctions of the hamiltonian:

$$
\hat{H}_{n}(p)=E_{n} \quad{ }_{n}(p) .
$$

Equation (5.6) is also called stationary equations of motion (stationary Schrödinger equation). Equation (5.5) shows time dependence of stationary states. Every wave function can be presented as a linear combination of stationary states:

$$
=\sum_{n} a_{n} \exp \left(-\frac{i}{-} E_{n} t\right){ }_{n}(p)
$$

where squared expansion coefficients $\left|a_{n}\right|^{2}$ denote probabilities of different energy values of the system. 
Below, though with rare exception, we will consider only ground or normal state of economy with least energy $E_{0}$ where its index 0 will be omitted. Probability distribution in stationary state is determined by ||$^{2}$ :

$$
|(p)|^{2}=|(p)|^{2} .
$$

That is, probability distribution does not depend on time.

If hamiltonian of economy represents a sum of two parts, e.g. a sum of hamiltonians of buyers' and sellers' subeconomies:

$$
\hat{H}=\hat{H}_{1}\left(p_{1}\right)+\hat{H}_{2}\left(p_{2}\right),
$$

where one of them comprises only price coordinates $p_{1}$, and the other only price coordinates $p_{2}$, so eigenfunctions of Hamilton operator of the entire economy can be written in the following form:

$$
\begin{gathered}
{ }_{12}\left(p_{1}, p_{2}\right)={ }_{1}\left(p_{1}\right) \quad{ }_{2}\left(p_{2}\right), \\
E_{12}=E_{1}+E_{2}, \\
\hat{H}_{12}\left(p_{1}, p_{2}\right)=E_{12} \quad{ }_{12}\left(p_{1}, p_{2}\right), \\
\hat{H}_{1}\left(p_{1}\right){ }_{1}\left(p_{1}\right)=E_{1} \quad{ }_{1}\left(p_{1}\right), \\
\hat{H}_{2}\left(p_{2}\right){ }_{2}\left(p_{2}\right)=E_{2} \quad{ }_{2}\left(p_{2}\right) .
\end{gathered}
$$

So ${ }_{1}$ and $E_{1}$ are eigenfunctions and eigenenergies of buyers' subeconomies, 2 and $E_{2}$ are eigenfunctions and eigenenergies of sellers' subeconomies. It is evident, that in this case we consider interaction between buyers and sellers insignificant. To avoid misunderstandings we remind that though we use the word 'energy' for economic systems, this notion is not related anyhow to physical systems and has a different dimension as compared to the real one. The notion is only used to make a parallel between studied economy and physical prototype more convenient. The essence of energy in economic theory is to be revealed in the future. So if (5.10) takes place, then

$$
\left.{ }_{12}\left(p_{1}, p_{2}\right)\right|^{2}=\left|{ }_{1}\left(p_{1}\right)\right|^{2}\left|{ }_{2}\left(p_{2}\right)\right|^{2}=d\left(p_{1}\right) s\left(p_{2}\right),
$$

where probability distribution of buyers $d(p)$ and sellers $s(p)$ is the following according to definition:

$$
d\left(p_{1}\right) \equiv\left|{ }_{1}\left(p_{1}\right)\right|^{2}, s\left(p_{2}\right) \equiv\left|{ }_{2}\left(p_{2}\right)\right|^{2} .
$$

We consider a priori that the probability of arranging a deal at $p$ price $f(p)$ is a product of these probabilities at equal values of price coordinates, that is, when $p_{1}=p_{2}$ :

$$
f(p)=d(p) s(p) .
$$


This formula will be used below.

Let us notice that quantum mechanics of atoms, molecules and solids, to put it more precisely, atomic theory and quantum chemistry of molecules and solids [6-8], has gained a great experience in computer-based solutions of Schrödinger equations and can be efficiently used for solutions of equations of motion of quantum economies. It makes sense to use this unique opportunity as much as possible.

\subsection{Economy with a single buyer and a single seller}

Let us go further and assume that if there is an economy with a single commodity (the case of one-dimensional price space), hamiltonian of a single buyer and a single seller economy with price coordinates $p_{i}(i=1,2)$ formally looks like hamiltonian of physical system of two material particles in physical space (physical prototype of economy), namely:

$$
\hat{H}=-\frac{2}{2 m_{1}} \frac{\partial^{2}}{\partial^{2} p_{1}}-\frac{2}{2 m_{2}} \frac{\partial^{2}}{\partial^{2} p_{2}}-V_{12}\left(p_{1}, p_{2}\right)-U_{1}\left(p_{1}\right)-U_{2}\left(p_{2}\right) .
$$

In equation (5.15) $m_{1}$ and $m_{2}$ are certain constants (a priori unknown) of the buyer and the seller. These constants for physical prototype are called masses but, of course, they have nothing to deal with masses for economy. They have different dimension and can only be determined by experiment. Operators $\frac{\partial^{2}}{\partial^{2} p_{1}}$ and $\frac{\partial^{2}}{\partial^{2} p_{2}}$ are second-order operators of differentiation with respect to price coordinates. Potential $V_{12}\left(p_{1}, p_{2}\right)$ is designed to describe the interaction between the buyer and the seller, and $U_{1}\left(p_{1}\right)$ and $U_{2}\left(p_{2}\right)$ are introduced into theory to describe the influence of external environment on economic agents. With the hamiltonian selected by this means, equations of motion of our economy have the following form:

$$
\begin{gathered}
\left(-\frac{2}{2 m_{1}} \frac{\partial^{2}}{\partial^{2} p_{1}}-\frac{2}{2 m_{2}} \frac{\partial^{2}}{\partial^{2} p_{2}}-\right. \\
\left.=V_{12}\left(p_{1}, p_{2}\right)-U_{1}\left(p_{1}\right)-U_{2}\left(p_{2}\right)\right){ }_{12}\left(p_{2}\right) .
\end{gathered}
$$

If we neglect the interaction between the buyer and the seller in comparison with the influence of external environment, then the equations of motion are divided into a system of two independent equations: 


$$
\begin{aligned}
& \int\left(-\frac{2}{2 m_{1}} \frac{\partial^{2}}{\partial^{2} p_{1}}-U_{1}\left(p_{1}\right)\right) \quad{ }_{1}\left(p_{1}\right)=E_{1} \quad{ }_{1}\left(p_{1}\right), \\
& \left\{\left(-\frac{2}{2 m_{2}} \frac{\partial^{2}}{\partial^{2} p_{2}}-U_{2}\left(p_{2}\right)\right) \quad{ }_{2}\left(p_{2}\right)=E_{2} \quad{ }_{2}\left(p_{2}\right)\right. \text {; } \\
& \left\{\begin{array}{c}
{ }_{12}\left(p_{1}, p_{2}\right)={ }_{1}\left(p_{1}\right){ }_{2}\left(p_{2}\right), \\
E_{12}=E_{1}+E_{2} .
\end{array}\right.
\end{aligned}
$$

Within the approximate self-consistent field approach [2], interaction $V_{12}$ can be also considered to get an analogous system of related equations:

$$
\left\{\begin{array}{lll}
\left(-\frac{2}{2 m_{1}} \frac{\partial^{2}}{\partial^{2} p_{1}}-U_{1}\left(p_{1}\right)-\widetilde{V}_{12}\left(p_{1}\right)\right) & { }_{1}\left(p_{1}\right)=E_{1} \quad{ }_{1}\left(p_{1}\right), \\
\left(-\frac{2}{2 m_{2}} \frac{\partial^{2}}{\partial^{2} p_{2}}-U_{2}\left(p_{2}\right)-\widetilde{V}_{21}\left(p_{2}\right)\right) & { }_{2}\left(p_{2}\right)=E_{2} \quad{ }_{2}\left(p_{2}\right) .
\end{array}\right.
$$

In equations (5.21) and (5.22) modified potentials $\widetilde{V}_{12}\left(p_{1}\right)$ and $\widetilde{V}_{21}\left(p_{2}\right)$ depend on solutions ${ }_{1}\left(p_{1}\right)$ and ${ }_{2}\left(p_{2}\right)$. All the equations obtained above are generalized for systems consisting of many buyers and sellers in an obvious manner.

Quantum mechanics and quantum physics have gained a great experience in calculating such equations of different level of mathematical complexity, and this experience greatly exceeds the one gained by classical mechanics, especially for many-particle systems. This is another argument in favor of a wider use of methods of quantum modeling compared to those of classical modeling. This experience is an efficient and, roughly speaking, a cost-free way to be used at the first stage of developing adequate physical models by selecting different types of potentials $U$ and interactions $V$, and by fitting solutions to the relevant experimental data regarding real economy. The same is true for finding parameters of economies and $m_{i}$.

However, we are able to say something about the type of probability distributions, the potential of interaction between the buyer and the seller, and about potentials of external environment using the properties of normal wave functions well-studied in quantum mechanics (we consider that they are well described as normal wave functions of a quantum anharmonic oscillator). Figures 5.1 and 5.2 present the assumed types of probability distributions. 
To get other general results we will give a more detailed consideration to probability distribution properties along with demand and supply functions related to them.

\subsection{Probability distribution of conducting transactions by sellers and buyers}

We will try to answer the question on how probability distributions of quantum economy are connected with empirical data, in the first place with demand and supply functions that can be basically measured by methods of empirical economics. Some assumptions are needed here too. Let us start from an elementary economy, with a single buyer and a single commodity. We assume a priori that the buyer tends to buy some goods for a sum of money $D_{0}$ and intends to arrange a deal in the market with a previously specified probability distribution $d(p)$ that he will make a purchase transaction at the price $p$. The experience in calculations of stationary states in quantum mechanics and common sense show that a typical probability distribution function for the buyer $d(p)$ 'must' have the form as in Fig. 5.1: at zero, at infinity and in the negative prices region it equals zero; it is evident that it has one maximum at the most probable purchase price $p_{d}^{m}$.

For the analogous situation with a single seller and a single commodity we consider a priori that dynamics of the system or seller's behavior in the market is completely determined by a desire to sell some goods for a sum of money $S_{0}$. Let us assume further that the probability that he will sell his goods at price $p$ is described by probability distribution function $s(p)$. The experience in quantum calculations and common sense show that a typical distribution $s(p)$ behaves similarly to distribution $d(p)$ (see Fig. 5.2). When $p \leq 0$ and $p \rightarrow+\infty$ the function tends to zero, and in the positive prices region has, as we may consider, a single maximum at the most probable sale price $p_{m}^{s}$.

Summing up, we may say that the suggested quantum or probabilistic approach describes buyers' and sellers' behavior in the market as of thinking, evaluating, hesitating and wavering people, that is homo oscillans (a wavering man): such a person or subject does not feel sure what price he should trade at in the market but there is a probability distribution what price the transaction should be made at.

\subsection{Demand and supply functions}

Further, we determine the buyer's demand function $D(p)$ and the seller's supply function $S(p)$ as products of distribution functions $d(p)$ 
and $s(p)$ by the total demand $D_{0}$ and the total supply $S_{0}$ respectively. Formally, all the above stated has the following form:

$$
\begin{aligned}
& D(p)=D_{0} d(p), \quad S(p)=S_{0} s(p) ; \\
& \int_{-\infty}^{\infty} D(p) d p=D_{0}, \quad \int_{-\infty}^{\infty} S(p) d p=S_{0} .
\end{aligned}
$$

It is natural that the form of these functions is congruent to the form of correspondent probability distributions (see Figs. 5.1 and 5.2). As it is seen in the figures, these functions behave in the medium prices region like straight lines which are usually used in neoclassical economic theory to show the demand and supply functions. Slope of the demand function is negative (as it is according to the empirical law of demand $[4,9,10]$ ), and slope of the supply function is positive (as for the empirical law of supply $[9,10])$.

\subsection{Probabilistic mechanism of determination of a market price}

At the moment of describing the model we have the elementary economic system or economy with the single buyer with a demand function $D(p)=D_{0} d(p)$ and the single seller with a supply function $S(p)=S_{0} s(p)$. So the question is: what is the price of a transaction between them? Or in other words, what price will the transaction be made at? We consider that the mechanism of making transactions and the mechanism of market pricing has a probabilistic nature: sales transactions can be made at any price but with a different probability. We consider it natural that if the probability of making a purchase by the buyer at price $p$ equals $D(p)$ and the probability of making a sale by the seller at price $p$ equals $S(p)$, then the probability that a purchase-sale transaction will be effected at price $p$ is equal to $F(p)$ and is a product of the purchases and sales probabilities:

where

$$
F(p)=D(p) S(p)=D_{0} S_{0} f(p),
$$

$$
f(p)=d(p) s(p) .
$$

It is evident that if one of the functions $D(p)$ or $S(p)$ represents rather a narrow probability distribution, then total probability distribution $F(p)$ will be narrow as well. In this case, a market price can be denoted by price $p_{m}$ at which the probability to make a purchase-sale transaction is maximal (the market price can be also regarded as a weighted average 
$\int_{-\infty}^{+\infty} F(p) p d p$

price $\bar{p}=\frac{-\infty}{+\infty}$ but it will make no difference because both prices

$$
\int_{-\infty}^{+\infty} F(p) d p
$$

are similar).

It is easy to show that as functions $F(p)$ and $f(p)$ are congruent (see (5.25)), they have their maximum at one and the same $p_{m}$. Thus, we may conclude that market price explicitly depends neither on total demand $D_{0}$ nor on total supply $S_{0}$. Now we see that a market price in this quantum model is determined only by behavior peculiarities of the demand and supply functions. All above stated is graphically presented in Fig. 5.3 where it is clearly seen how a market price $p_{m}$ is formed as a compromise value between the buyer's low price $p_{m}^{d}$ and the seller's high price $p_{m}^{s}$, as a result of trading, that is by negotiating and exchange of information between the buyer and the seller.

Another problem of interest is what quantity of goods will be really bought or sold in the market at certain demand and supply functions, however, this task is beyond the scope of the present model and its solution will depend on special rules of market activities specified out of the present model. As it is accepted in neoclassical economic theory [4, 9, 10] we may assume as one of possible rules that total monetary trade volume in the market will be determined by the minimum of two values: $D\left(p_{m}\right)$ and $S\left(p_{m}\right)$ (this is a quantity of sold and bought goods by definition) multiplied by price $p_{m}$ :

$$
P=\min \left\{D\left(p_{m}\right), S\left(p_{m}\right)\right\} p_{m} .
$$

We may also assume that the market makes all possible claimed transactions at price $p_{m}$, so we can determine the monetary trade volume by the following formula:

$$
P=\min \left\{D_{0}, S_{0}\right\}
$$

Fig. 5.3. Graphs of probabilistic mechanism for formation of market price $p_{m}$.

$$
1-S(p), 2-D(p), 3-F(p) .
$$

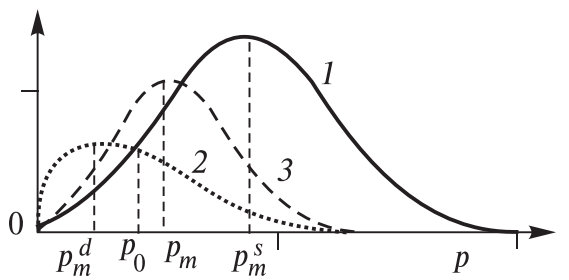




\subsection{Market price and demand and supply elasticities}

There are certain observations in scientific literature about relation between a market price and demand and supply elasticities but the type of this relation is not expressed explicitly $[9,10]$. Within the framework of the quantum model such a relation is found trivially. It is known that derivative of function equals zero at the maximum point. As regards our case it means that derivative function $F(p), d F(p) / d p \equiv F^{\prime}(p)$, must equal zero at maximum point $p_{m}$. With the help of simple calculations we can find that required price $p_{m}$ is a solution of equation

$$
\frac{D^{\prime}(p)}{D(p)} p=-\frac{S^{\prime}(p)}{S(p)} p,
$$

where demand and supply elasticities are on the left and on the right $E D(p)$ and $E S(p)$ respectively:

$$
E D(p) \equiv \frac{d D(p)}{d p} \frac{p}{D(p)} \text { and } E S(p) \equiv \frac{d S(p)}{d p} \frac{p}{S(p)} .
$$

So we have obtained rather an interesting result, that is, that maximum probability of transaction is achieved at that price $p_{m}$ when demand and supply elasticities are equal in absolute value but opposite in sign. This is illustrated in Fig. 5.4.

\subsection{System comprising arbitrary numbers of sellers and buyers}

Let us consider an economic system comprising an arbitrary number of different buyers $N$ and an arbitrary number of sellers $M$. For such

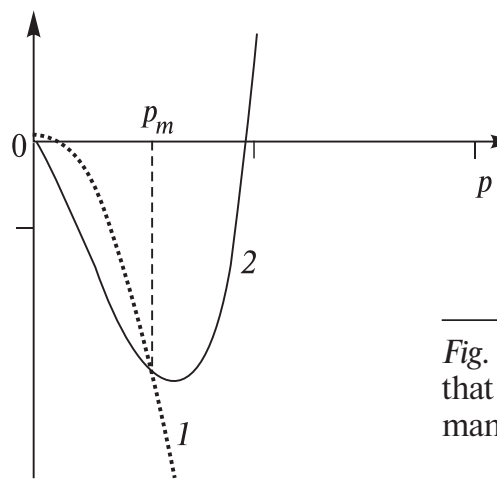
kind of economy we make several assumptions analogous to those made for elementary economies, namely:

1. Economy is in the ground or normal stationary state, where there are no strong external perturbations depending on time.

Fig. 5.4. Graphs plotted for the result of the theory that market price $p_{m}$ is at the intersection of demand (1) and supply (2) elasticity curves (the last one has an opposite sign). 
2. Buyers' demand function $D(p)$ and sellers' supply function $S(p)$ are directly proportional to probability distributions $d(p)$ and $s(p)$ of conducting sales transactions at price $p$ by buyers and sellers in the market:

$$
\begin{gathered}
D(p)=D_{0} d(p) \text { and } S(p)=S_{0} s(p) ; \\
\int_{-\infty}^{+\infty} d(p) d p=1, \int_{-\infty}^{+\infty} s(p) d p=1 ; \\
\int_{-\infty}^{+\infty} D(p) d p=D_{0}, \int_{-\infty}^{+\infty} S(p) d p=S_{0},
\end{gathered}
$$

where $D_{0}$ and $S_{0}$ are total demand and supply in the market, and demand function $D(p)$ and supply function $S(p)$ represent probability distribution of conducting purchases and sales transactions at price $p$ respectively.

3. Probability that the transaction will be really conducted at the price $p, F(p)$ is a product of two probabilities:

$$
F(p)=D(p) S(p) .
$$

Reasoning analogous to the previous one made for the elementary system comprising a single buyer and a single seller shows that sales transactions are conducted in the market mainly at the price range near $p_{m}$ corresponding to the maximum probability of function $f(p)=d(p) s(p)$ or to the maximum of product of the demand and supply functions: $F(p)=D(p) S(p)$.

Using the same method we can show that point $p_{m}$ corresponds to the point of intersection of the demand elasticity function $(E D(p))$, and the supply elasticity function, taken with the opposite sign $(-E S(p))$.

The above graphically described situation is presented in the same figures as for the case with a single buyer and a single seller economy (see Figs. 5.1-5.4).

It is notable that if slopes of the demand and supply functions approximately equal to each other (to be more precise, if $D^{\prime}(p) \cong-S^{\prime}(p)$, e.g. $D(p)$ and $S(p)$ are linear functions with slopes being equal in value but opposite in sign), then point $p_{m}$ coincides with the point $p_{0}$ of intersection of demand $D(p)$ and supply $S(p)$ functions:

$$
D\left(p_{0}\right)=S\left(p_{0}\right) \text {. }
$$

Hence, in this case transactions will be conducted in the market mainly near intersection $p_{0}$ of the demand and supply functions. This point is often regarded as a point of equilibrium in neoclassical economic theory and this price determines in this theory all transactions 
in the market with monetary trade volume $P$ which can be calculated by the following formula:

$$
P=\min \left\{D\left(p_{0}\right), S\left(p_{0}\right)\right\} \times p_{0} .
$$

\subsection{The additivity rule for demand and supply functions}

For practical use we can introduce one more assumption into the model.

4. If there is a relative weak interaction $V$ between buyers and sellers, then demand and supply functions for the whole system may be calculated as a sum of the demand and supply functions of individual buyers and sellers:

$$
\begin{aligned}
& D(p) \cong \sum_{i=1}^{N} D_{i}(p)=\sum_{i=1}^{N} D_{0}^{i} d_{i}(p), \\
& S(p) \cong \sum_{j=1}^{M} S_{j}(p)=\sum_{j=1}^{M} S_{0}^{j} S_{j}(p) .
\end{aligned}
$$

\subsection{Excited states of quantum economy}

We have confined ourselves to consideration of only ground or normal state of quantum economy so far. However, we know that apart from a normal state there are infinitely many excited states with energy $E_{n}$, a greater energy compared to that of a normal state, see equations of motion (5.4)-(5.6). The fact that such excited states may exist has a great significance. At present we know about excited states only that their wave functions have one or more nodes, thus, probability distribution has two or more maximums, that is why the above given definition of demand and supply functions hardly makes sense for excited states or it should be somehow generalized. Intuitively it is also evident that excited states of economy are states of less order and greater chaos in economy, therefore, of its lower efficiency. It is also clear that economy may transfer from normal state to excited states and back under strong time-dependent perturbations of the environment $U(p, t)$. It is known from equations of motion that if at some point of time $t_{0}$ economy was in normal state and the environmental influence was reduced to constant potential $U(p)$, then economy will be in normal state for an infinitely long period of time.

So, it results from quantum theory of economy that the government is not recommended making drastic changes in its economic policy to reduce the possibility to transfer the economy from the normal state to 
an excited state, that is, to less efficient state. This conclusion of quantum theory of economy is consistent with the viewpoint of neoclassical economic theory that intervention by the government into economy is harmful as it reduces the economy's efficiency $[4,9,10]$.

So, this is a true fact. But it is quite another matter when economy is already in an excited state. In this case, according to quantum theory it is necessary for the government to cause time-dependent strong and abrupt perturbation $U(p, t)$ to make economy gradually return from the excited state to the normal state. Market economy itself cannot make it with its own internal powers (i.e. with $V(p)$ potentials). In other words, we may conclude from quantum theory that spontaneous market forces are not enough in principle to make economy transfer from the excited state to the normal one, that is, recover from recession and begin to improve. In this case, the role of government and its interference in economy is the barest necessity and a benefit for the whole economy. The problem lies only in the 'right' diagnosis of economy, 'right' time and 'right' measures (that is, in choosing a suitable perturbation $U(p, t)$ ). 


\section{Conclusion}

In this paper we have developed physical, classical and quantum models of some economic systems by drawing a strict analogy with a theory of analogous physical systems. There are some advantages in these models. On the one hand, they make possible to simultaneously consider influence exerted by the interaction of market subjects on the economic system (a major subject in neoclassical economic theory) and interaction of the environment, government, society and other institutions with economic agents (subject of investigation by institutional economic theory), and on the other hand, to get equations of motion of economy that allow describing evolution of the economic system in time (paradigm of evolutionary economics). Besides, the equations obtained can be calculated by computers using insignificantly modified programs for calculation of electronic structure of atoms and molecules, in other words, methods of atom theories and quantum chemistry of molecules and solids [6-8].

We suppose that we have obtained only a starting or initial approximation for more exact classical and quantum models that may appear after more complete and continuous investigations in this field. As it often happens after discovering new approaches and theories, more questions occur than before. First of all, it is a question on applicability limits of such a physical modeling of complicated economic systems, a question on economic essence of parameters and potentials of models, etc. We hope to clarify all these questions in the future. 


\section{References}

1. Landau L.D., Lifshitz E.M. Theoretical Physics, 1, Mechanics, Fizmatlit, Moscow (2002).

2. Landau L.D., Lifshitz E.M., Theoretical Physics, 3, Quantum Mechanics. Nonrelativistic Theory, Fizmatlit, Moscow (2002).

3. Intriligator M., Mathematical Methods of Optimization and Economic Theory, Airis-press, Moscow (2002).

4. Vorkuev B.L., Models of Macro-and Microeconomics, Moscow (1999).

5. J. von Neumann and O. Morgenshtern, Theory of Games and Economic Behavior, Nauka, Moscow (1970).

6. Kondratenko A.V., Mazalov L.N., Topol' I.A., Highly-Excited States of Molecules, Nauka, Novosibirsk (1982).

7. Kondratenko A.V., Neumann K.M., Quantum Chemistry and Spectroscopy of Highly-Excited States. Coordination Compounds of Transition Metals, Nauka, Novosibirsk (1990).

8. Kondratenko A.V., Cederbaum L.S., Phys. Rev., B43, 10595 (1991).

9. Sio K.K., Managerial Economics, Infra-M, Moscow (2000).

10. Frank R.Kh., Microeconomics and Behavior, Infra-M, Moscow (2000). 


\section{Contents}

1. Introduction . . . . . . . . . . . . . . . . . . . . . . . 3

2. Main elements of the physical models f . . . . . . . . . . . . . 6

3. Price space . . . . . . . . . . . . . . . . . . . . . . . . . . . . . . . . . 7

4. Classical economy . . . . . . . . . . . . . . . . . . . . . . . . . . . . . . 9

5. Quantum economy . . . . . . . . . . . . . . . . . . . . . . . . 14

5.1. Time-dependent equations of motion of quantum economy 14

5.2. Stationary equations of motion . . . . . . . . . . 16

5.3. Economy with a single buyer and a single seller . . . . . . 18

5.4. Probability distribution of conducting transactions by sellers and buyers . . . . . . . . . . . . . . . . . . 21

5.5. Demand and supply functions . . . . . . . . . . . 21

5.6. Probabilistic mechanism of determination of a market price. . 22

5.7. Market price and demand and supply elasticities . . . . . . 24

5.8. System comprising arbitrary numbers of sellers and buyers . . 24

5.9. The additivity rule for demand and supply functions . . . . . 26

5.10. Excited states of quantum economy . . . . . . . . . . 26

6. Conclusion . . . . . . . . . . . . . . . . . . . . . . . . . . . . . 28

References . . . . . . . . . . . . . . . . . . . . . . . . . . . . . . . . 29 